\title{
Indirect Searches for Dark Matter with IceCube
}

\author{
Juan Antonio Aguilar Sánchez ${ }^{1, *}$ for the IceCube Collaboration** \\ ${ }^{1}$ Université Libre de Bruxelles
}

\begin{abstract}
The nature of dark matter remains one of the unsolved questions in modern cosmology and to understand its properties different experimental avenues are being explored. Indirect searches make use of the annihilation or decay products of dark matter as tracers to prove its existence. Unlike direct detections methods, indirect searches do not require specialized detectors as existing astro-particle experiments and facilities can be used to search for signatures of dark matter. Among the decay and annihilation products, neutrinos offer a unique way to search for dark matter since their low cross-section makes them capable of escaping from environments in which gamma rays will be absorbed, like the Sun or the Earth. The IceCube neutrino telescope is not only an excellent astro-particle detector, it also has lively program on dark matter searches with very competitive and complementary results to direct detection limits. These proceedings review the latests results of IceCube regarding the indirect search of dark matter with neutrinos.
\end{abstract}

\section{Introduction}

The IceCube observatory is a neutrino telescope located at the geographic South Pole consisting of an array of 5,160 photomultipliers tubes (PMTs) distributed along 86 strings and deployed between 1.5-2.5 km deep within the Antarctic ice cap [1]. IceCube neutrino detection principle is based on the observation of Cherenkov light along the path of secondary particles produced in neutrino interactions with the matter surrounding the detector. The main scientific goal of IceCube is the identification of neutrino sources and those responsible of the cosmic-ray emission. Unlike cosmic rays, neutrinos can travel undeflected through the cosmos, bringing directional information about the origin of their production. In addition, compared to gamma rays that interact with the Cosmic Microwave Background (CMB) and the infrared starlight background, neutrinosínteraction length is comparable to the estimated size of the observable Universe.

\section{Indirect searches of dark matter}

Several experimental data suggest the existence of a significant contribution of non-visible matter in our Universe. This evidence comes from a variety of observations such as the study of the rotational dynamics of spiral galaxies, the virialization of galaxy clusters, the observation of gravitational lensing, and the analysis of the power spectrum of the CMB as

\footnotetext{
*e-mail: analysis@icecube.wisc.edu

**http://icecube.wisc.edu
} 
well as the Big Bang nucleosynthesis. Understanding the nature of dark matter is one of the long-standing problems in modern physics. Indirect searches aim at the identification of dark matter by looking at the products of potential decays and/or annihilations. Among these products, neutrinos could provide a unique proof to the existence of dark matter. In the following we summarize the results of different analyses performed with IceCube data.

\subsection{Galactic and extra-Galactic dark matter searches}

Over-fluctuations of the diffuse dark matter density developed together with the structure formation in our Universe. The study of structure formation from N-body simulation, as well as from direct observation of the kinematics of stars in galaxies, predicts an excess of dark matter at the center of galaxies. The inner region of our Galaxy is therefore one of the most interesting regions to search for the products of annihilation of dark matter. These searches, however, depend strongly on the astrophysical inputs, in particular on the exact shape of the dark matter density profile. Figure 1 left shows the upper limits on the velocity averaged dark matter annihilation cross-section from different analyses in IceCube considering different event selections [2]. It is also possible to search for decay products rather than annihilation. Kinematically dark matter could decay as long as its lifetime is longer than the age of the Universe. Unlike with annihilation, the event rate is now proportional to the density of dark matter, and not density squared, which means that over-densities such as the inner region of the Galaxy have a lesser impact on the decay rates and the extra-galactic contribution is no longer negligible. For decaying dark matter the results are presented in terms of lower limits of the dark matter decay lifetime. Two independent statistical methods have been applied in IceCube to search for a contribution of decaying dark matter to the astrophysical neutrino spectrum [3]. The results are compatible with an astrophysical origin only and lower limits of the decay lifetime have been produced (see Fig. 1 right).
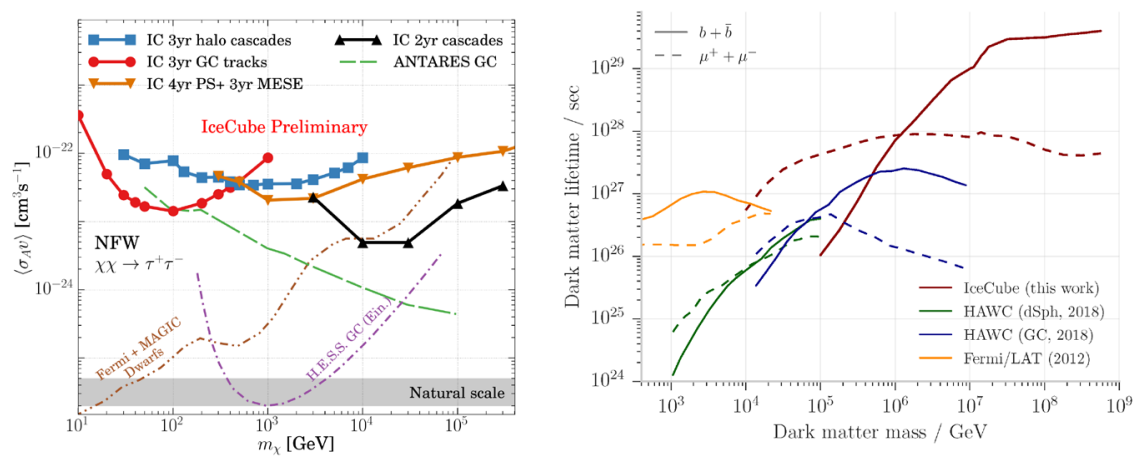

Figure 1. Left: Upper limits on the $\left\langle\sigma_{A} \mathrm{v}\right\rangle$ as function of the dark matter mass for the annihilation channel $\chi \chi \rightarrow \tau \tau$ assuming the Navarro-Frenk-White halo profile [2]. Right: Lower limits on the dark matter decay lifetime [3].

\subsection{Dark Matter annihilation from the Sun and Earth}

Assuming that dark matter particles interact with Standard Model particles, they could scatter with nucleons of the Sun or Earth losing enough energy to be gravitationally captured. This process implies that, over time, an excess of dark matter accumulates at the center of these objects. Like in the case of dark matter from the Galactic Center, the over-density would 
enhance the dark matter self-annihilation. Given the high matter density of the Sun and Earth, among the annihilation products only neutrinos will escape to the surface, making these sources only accessible with neutrino detectors. Contrary to the Galactic Center, the capturing process inside celestial bodies only depends on the local dark matter density, whose estimate is better constrained than the overall density profile. However, the capturing will be influenced by the local velocity distribution of dark matter halo particles. The capturing process reaches an equilibrium when the capturing rate equals the annihilation rate. For values of $\sigma_{\chi p}^{S I} \sim 10^{-43} \mathrm{~cm}^{-2}$ the time needed to reach equilibrium is of the order of $10^{11}$ years in the case of the Earth, while for the Sun the time for equilibrium is smaller than the age of the solar system. Therefore, the equilibrium condition applied to the Sun makes it possible to infer an upper limit on $\sigma_{\chi p}$. IceCube results on the searches for dark matter from the Sun and the Earth are shown in Fig. 2. For the case of the Earth an annihilation cross-section needs to be assumed in order to derive limits in the spin-independent dark matter-proton cross-section. A typical assumed value is the thermal relic cross section, $3 \times 10^{-26} \mathrm{~cm}^{3} \mathrm{~s}^{-1}$.
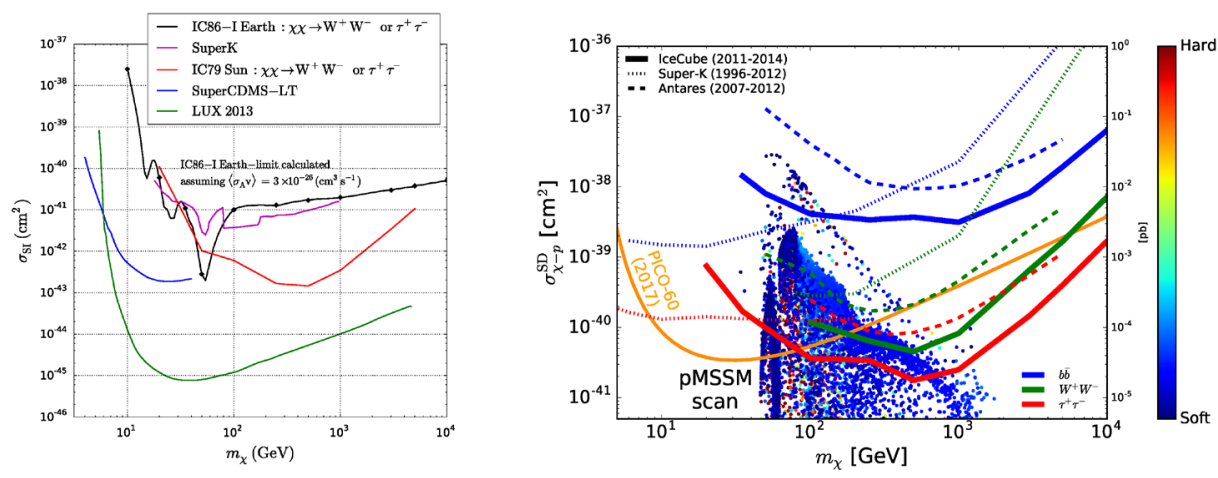

Figure 2. Left: Limits on the spin-independent dark matter-proton cross-section $\sigma_{\chi-p}^{S I}$ as a function of the dark matter mass from the Earth [4] assuming $\left\langle\sigma_{A} v\right\rangle=3 \times 10^{-26} \mathrm{~cm}^{-3} \mathrm{~s}^{-1}$. Right: Limits on the spin-dependent dark matter-proton cross-section $\sigma_{\chi-p}^{S D}$ from the Sun compared to results from other neutrino detectors and direct detection experiments [5].

\subsection{Scattering of dark matter}

The directional distribution of the astrophysical neutrinos detected by IceCube is compatible with an isotropic distribution. The isotropy and the result of a dedicated galactic plane correlation analysis suggest that the astrophysical neutrinos are extra-Galactic. If dark matter can couple to neutrinos, this means that the directional distribution of neutrinos travelling throughout our Galaxy can be influenced by dark matter. In particular, a suppressed flux towards the densest part of our Galaxy could be visible. Using 103 high-energy starting events of astrophysical neutrinos an analysis was carried out looking for this deficit towards the center of the Galaxy assuming two simplified models: one in which dark matter is assumed to be a fermion and the mediator is a vector, and a second model in which dark matter is assumed to be a vector while the mediator is a fermion. The results of this analysis showed that no flux suppression is present towards the Galactic Center and therefore limits on the dark matter neutrino coupling were derived. These limits are shown in Fig. 3 for the two models described above. 

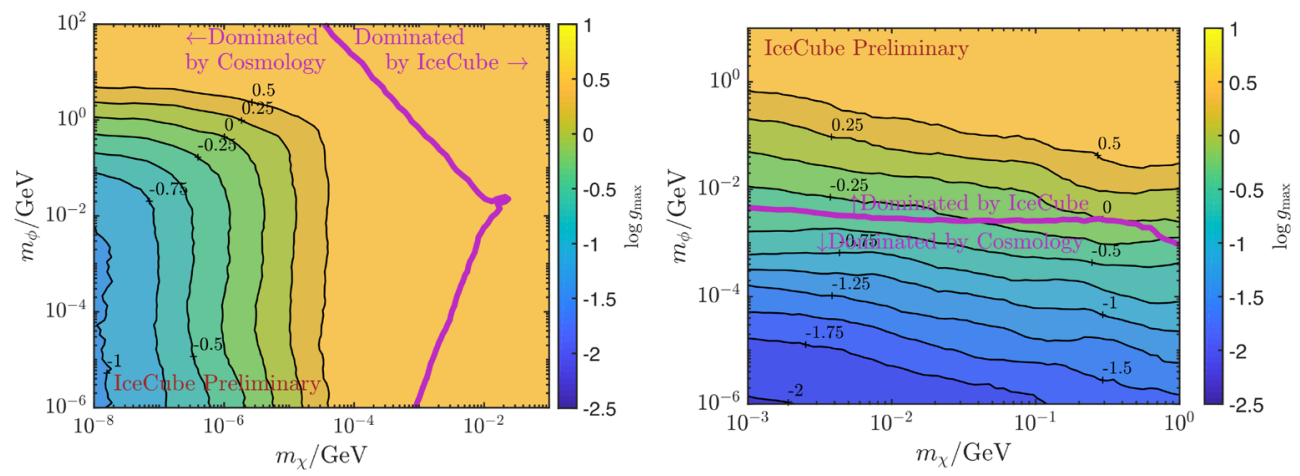

Figure 3. Limits on the maximum coupling as function of mediator and dark matter mass. The color scale shows the maximum allowed coupling. The region signal labeled "dominated by IceCube" means that in this region IceCube bounds are the strongest. The region labelled "dominated by Cosmology" indicates that in that area the cosmological bounds are stronger. Left: Limits for the scalar-fermion model. Right: Limits for the fermion-vector model [6].

\section{Conclusions}

Dark matter remains elusive despite the multiple experimental and phenomenological efforts to unravel its nature. A signal from indirect detection could be easily misinterpreted as having an astrophysical origin and therefore multiple observations from other searching strategies are required to obtain a meaningful discovery. Indirect dark matter detection through neutrinos provides a complementary way to explore dark matter due to the different expected backgrounds and the fact that neutrinos can escape from dense objects such the Sun or the Earth where dark matter would annihilate. IceCube has a very active program on indirect detection of dark matter and is able to set the best limits in spin dependent dark matter-proton cross-section for some regions of the parameter space.

\section{References}

[1] IceCube collaboration, JINST 12, P03012 (2017)

[2] IceCube collaboration, Eur. Phys. J. C77, 627 (2017)

[3] IceCube collaboration, Eur. Phys. J. C78, 831 (2018)

[4] IceCube collaboration, Eur. Phys. J. C77, 82 (2017)

[5] IceCube collaboration, Eur. Phys. J. C77, 146 (2017)

[6] IceCube collaboration. Neutrino 2018. doi:10.5281/zenodo.1300506 